\title{
Proceso de Bolonia (IV): currículo o plan de estudios
}

\author{
Joan Prat-Corominas, Albert Oriol-Bosch
}

'The curriculum is a sophisticated blend of educational strategies, course content, learning outcomes, educational experiences, assessment, the educational environment and the individual student.'

R.M. Harden (2001)

\section{Introducción}

La creación del Espacio Europeo de Educación Superior (EEES), conocido como proceso de Bolonia, hunde sus raíces en tres conceptos derivados de la construcción europea. El primero es la potenciación de la ciudadanía europea a través del intercambio de universitarios entre países diferentes, que está en la base de los programas Erasmus y Sócrates. El segundo es el concepto de la sociedad y la economía basadas en el conocimiento a que aspira Europa, basados en la formación, la investigación y el desarrollo como pilares estratégicos. La declaración de la Sorbona en 1998, punto de partida de todo el proceso, explicita la necesidad de colocar las universidades europeas entre las más relevantes del mundo con este fin. El tercero, las consecuencias del acuerdo de Schengen sobre la libre circulación de personas y títulos, impone una armonización de éstos.

El proceso de cambio curricular llevado a cabo en España como consecuencia de los acuerdos de Bolonia constituye la manifestación más evidente de la incorporación de la formación superior española al EEES. Todas las facultades de medicina de España -las que ya existían y las que han surgido en este proceso- han tenido que efectuarlo dentro del plazo previsto (2010).

Ha sido un esfuerzo importante que ha exigido muchas horas de trabajo a las comisiones institucionales creadas para ello. En las facultades ya existentes este proceso se repite tres lustros después de que se aprobara el plan de estudios que venían desplegando. Un plan de estudios que también entonces se puso en marcha simultáneamente en todas las universidades por voluntad de la autoridad reguladora.

Cuando aún está presente la fatiga institucional causada por el esfuerzo y los conflictos internos que el proceso ha conllevado, parece conveniente reflexionar sobre el concepto de plan de estudios o 'currículo' y la complejidad que dicho concepto encierra para analizar las consecuencias del proceso de Bolonia sobre los aspectos más fundamentales de los currículos españoles. Debemos tener presente la existencia de distintos currículos: el formal, el real y el evaluado, aun cuando el presente análisis se limita al currículo formal (Figura).

El currículo constituye un programa de múltiples actuaciones [1]. En el currículo formal se explicita lo que la institución enseña y lo que los alumnos deben aprender. Algunos lo han denominado 'currículo ficticio' [2]. Se trata del currículo normativo, el que se publicita en el Boletín Oficial del Estado. Define solamente una parte -menor según algunos autores- de todos los procesos de aculturación social y profesional del estudiante. El currículo real lo constituye todo lo que los alumnos han llevado a cabo realmente, en tanto que el currículo aprendido y evaluado es aquel en el que se recogen los logros alcanzados por los alumnos y son evaluados por la institución. Constituyen los resultados del aprendizaje que en la actualidad se definen como competencias adquiridas.

Tradicionalmente se conceptualiza el currículo como la descripción de contenidos, su secuenciación temporal y las cargas docentes asignadas a cada una de las unidades educativas (asignaturas, materias, bloques, módulos, etc.). Sin embargo, un currículo debe contemplar por lo menos cuatro elementos distintos: los contenidos, por supuesto, pero también los recursos de aprendizaje ofertados desde una estrategia educativa institucional, los resultados esperados del aprendizaje y las evaluaciones previstas para promover y documentar el logro por el alumno de los objetivos educativos previstos.
Facultad de Medicina; Universitat de Lleida (J. Prat-Corominas). Presidente de la Fundación Educación Médica (A. Oriol-Bosch).

E-mail:

joan.prat@mex.udl.cat; aoriolb@terra.es

(c) 2011 Educación Médica 
Figura. Los tres currículos (modificado de [1]).

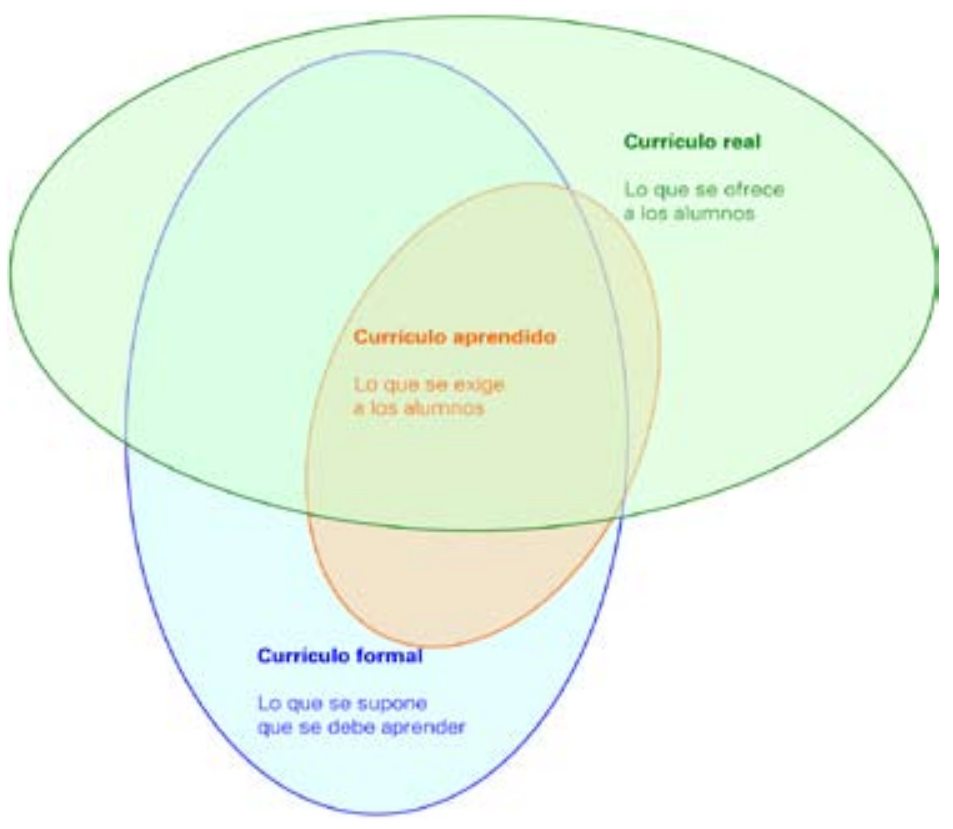

Tabla I. Elementos esenciales de un currículo.

\begin{tabular}{lcc}
\hline & \multicolumn{1}{c}{ Perspectiva } & Utilidad \\
\hline Contenidos & Lo que se debe tratar & Qué se debe conocer \\
\hline Recursos & Con qué elementos se cuenta & Cómo y con qué lograrlo \\
\hline Resultados & Qué debe adquirir el estudiante & Reconocer el logro \\
\hline Evaluaciones & Cómo se va a medir & Verificar el logro \\
\hline
\end{tabular}

Cada elemento pone de relieve una perspectiva distinta: la descripción de los contenidos se centra en lo que debe enseñarse, los resultados del aprendizaje definen lo que los alumnos deben haber aprendido, los recursos educativos explicitan cómo y donde pueden aprender los alumnos, y la evaluación, la forma en que éstos pueden valorar su aprendizaje y cómo serán valorados. Cada elemento curricular resuelve cuestiones diferentes del mismo proceso educativo desde una perspectiva distinta, siendo necesario abordarlas todas ellas de un modo coherente y equilibrado para alcanzar un currículo eficaz (Tabla I).
Si en el diseño curricular todo el empeño se ubica en la definición de contenidos, las cargas docentes que se les atribuyan y su posición en la secuenciación temporal, el resultado obtenido tenderá a reflejar los equilibrios de poder de los equipos docentes de la institución. Resulta pues indispensable abordar el diseño curricular desde una perspectiva estratégica contemplando tres premisas irrenunciables:

- El diseño curricular constituye una tarea colectiva.

- Debe contemplar coherentemente los cuatro elementos antes enumerados.

- Debe considerar siempre como un objetivo absoluto la obtención de un perfil adaptado a las necesidades del desempeño profesional (Tabla II).

Es menester, además, atender a las estrategias educativas y a la idoneidad de las oportunidades de aprendizaje así como a la evaluación no tan sólo con fines certificadores, sino también para conocer el éxito educativo del currículo y, por ende, la necesidad de mejorarlo. El proceso curricular debe desarrollarse según el modelo clásico de la calidad: diseñar, llevar a cabo, evaluar y rediseñar (ciclo de PDCA: Plan-Do-Check-Act) [3].

El currículo, a su vez, debe considerar los recursos educativos de todo orden (personales, infraestructurales, tecnológicos, de conocimiento, culturales, normativos...) [4] que están disponibles en la institución o se requieren para su implementación, y que condicionan las estrategias y metodologías utilizables. De esta forma, el diseño curricular constituye también una oportunidad de optimización en el uso educativo de los recursos institucionales disponibles. Por ello, el currículo hay que concebirlo no sólo como un catálogo de materias de las que se detallan los contenidos y como la secuenciación de actividades académicas o las cargas de trabajo para docentes y discentes o los objetivos educativos que deben alcanzarse. Comprende todo esto simultáneamente y mucho más, que se desprende de la misión de la institución, en el centro de la cual se encuentra siempre el servicio a la sociedad a través de los grandes objetivos institucionales, como la necesidad de dar respuesta a los requerimientos sociales percibidos y de hacerlo incorporando las estrategias educativas más efectivas.

\section{Modelos curriculares}

Existe en la actualidad un amplísimo abanico de currículos conducentes a la obtención del título de médico [5], lo que refleja una considerable diversidad de criterios estratégicos. Se pueden agrupar en 
Tabla II. Principios del diseño curricular.

Abordaje colectivo de la tarea

Definir los cuatro elementos básicos

Orientar al ejercicio competente de la profesión

modelos a partir de las estrategias principales que orientan su diseño. Una de las clasificaciones más reconocidas en la bibliografía [6,7] responde a las estrategias definidas en el modelo SPICES de Harden $[8,9]$. En esta clasificación se establecen cuatro grandes modelos curriculares, cada uno de ellos con numerosas variantes (Tabla III).

El modelo flexneriano, el más antiguo, utiliza estrategias educativas clásicas, y los más modernos, las estrategias más innovadoras. Así, el modelo flexneriano se centra en la información y en el profesor y se estructura en áreas de conocimiento (asignaturas), mientras que el más novedoso, el basado en presentaciones clínicas, se centra mayoritariamente en la resolución de problemas y en la participación del estudiante, se estructura en torno a un aprendizaje integrado y se desarrolla en base a situaciones clínicas. Cada uno de los modelos responde a unas prioridades o metas estratégicas que cada institución debe tener claramente definidas a la hora de iniciar el trabajo de planificación (Tabla IV).

Existen otras estrategias de diseño curricular, como puede ser la basada en la comunidad [10], que no se reflejan en esta clasificación.

El informe para la Carnegie Foundation sobre la formación de médicos en Norteamérica, de Abraham S. Flexner [11], se considera la primera propuesta curricular comprensiva para la formación de médicos de alcance mundial.

El modelo flexneriano se caracteriza por un diseño escalonado de la adquisición de conocimientos, de los más científicos a los más clínicos, asumiendo la cimentación de los segundos sobre los primeros. Su integración la debe hacer el propio estudiante. El objetivo principal es la aportación del conocimiento científico a la formación médica. Los currículos flexnerianos típicos se caracterizan por el fuerte peso de las áreas de conocimiento como estructura nuclear de las asignaturas y la tradicional división del currículo en dos ciclos, uno básico y otro clínico, lo que no acerca el conocimiento científico al clínico.

El diseño aditivo de Flexner ha propiciado la fragmentación del aprendizaje en compartimentos es-
Tabla III. Estrategias que caracterizan los principales modelos curriculares.

\begin{tabular}{lcccc}
\hline & $\begin{array}{c}\text { Flexneriano } \\
(1910)\end{array}$ & $\begin{array}{c}\text { Integrado } \\
(1957)\end{array}$ & $\begin{array}{c}\text { Basado en } \\
\text { problemas (1967) }\end{array}$ & $\begin{array}{c}\text { Presentaciones } \\
\text { clínicas (1995) }\end{array}$ \\
\hline $\begin{array}{l}\text { Centrada en el } \\
\text { profesor/estudiante }\end{array}$ & El profesor & Intermedio & El estudiante & Intermedio \\
\hline $\begin{array}{l}\text { Información/resolución } \\
\text { de problemas }\end{array}$ & Información & Información & $\begin{array}{c}\text { Resolución } \\
\text { de problemas }\end{array}$ & Intermedio \\
\hline \begin{tabular}{l} 
Disciplinas/integración \\
\hline
\end{tabular} & Disciplinas & Integrado & Integrado & Integrado \\
\hline
\end{tabular}

Tabla IV. Metas características de los principales modelos de diseño curricular.

\begin{tabular}{ll}
\hline Flexneriano & $\begin{array}{l}\text { Asegurar una amplia base de conocimientos científicos } \\
\text { Asegurar amplios conocimientos clínicos }\end{array}$ \\
\hline Integrado & $\begin{array}{l}\text { Asegurar el razonamiento científico del conocimiento clínico } \\
\text { Potenciar un perfil de investigador médico }\end{array}$ \\
\hline Por problemas & $\begin{array}{l}\text { Asegurar la creatividad y la capacidad crítica } \\
\text { Asegurar la capacidad de manejo de la información } \\
\text { Asegurar la capacidad de comunicación y trabajo en equipo }\end{array}$ \\
\hline Presentaciones & $\begin{array}{l}\text { Asegurar la adquisición de modelos de razonamiento clínico } \\
\text { Asegurar la capacidad de uso de los modelos en diferentes situaciones }\end{array}$ \\
\hline
\end{tabular}

tancos (mosaicismo), que no favorece la interrelación del conocimiento.

El desarrollo de los programas flexnerianos se halla profundamente influido por la idea de que los conocimientos científicos son la única herramienta imprescindible para el desarrollo de una actuación profesional competente. Este modelo no parece el más indicado para conseguir un razonamiento integrado, que constituye la base de la formación en competencias, por lo menos tal como se aplica en muchos centros.

El modelo flexneriano, sin embargo, aún prima actualmente en las facultades españolas y en general en los países del sur de Europa [12] por la consistencia del modelo con las estructuras orgánicas y los procesos organizativos de la actividad académica (departamentos, asignaturas, áreas de conocimiento).

Los currículos integrados lo pueden ser horizontal y verticalmente. La integración horizontal se expresa en esquemas curriculares ordenados por aparatos y sistemas, en tanto que la vertical incorpora las ciencias básicas a las clínicas y viceversa. Han aparecido en gran parte como respuesta a las limitaciones del modelo flexneriano. El primero de ellos fue elaborado a mediados de los años cincuenta por 
el Cleveland Clinic Lerner College of Medicine de la Case Western Reserve University (CWRU) en Cleveland (Ohio). La integración permite incrementar la relevancia de las ciencias básicas y de los contenidos científicos en las ciencias clínicas. Este modelo obliga a trabajar conjuntamente a los docentes básicos y clínicos, lo que permite seleccionar los contenidos de acuerdo a criterios de relevancia. Otra característica del modelo es el contacto temprano con la clínica como consecuencia de la integración.

Los conceptos y experiencias desarrollados en la CWRU iniciaron una etapa de potenciación de los currículos integrados como herramienta para la mejora docente a través del diseño curricular en nuevas facultades, como la Charité, en la refundida Universidad Humboldt de Berlín. De entre estos currículos integrados, un modelo que se encuentra en expansión, iniciado en los años noventa en esta última universidad y en el Tomas Jefferson Medical College de Filadelfia, es el currículo en espiral. Básicamente, el currículo en espiral consiste en un currículo integrado por sistemas en el que éstos se repiten dos o tres veces a lo largo del mismo. Así, en la primera aproximación al sistema, en los cursos iniciales, predominan los contenidos básicos ordenados por criterios clínicos de una fácil correlación básica. En la segunda vuelta de la espiral, la aproximación al sistema se hace desde una perspectiva clínica con las aportaciones básicas pertinentes. Finalmente, la tercera aproximación al sistema, cuando la hay, se lleva a cabo desde la actividad asistencial (durante el rotatorio o clerkship). La base que sostiene el carácter integrado de este modelo lo constituye la planificación conjunta del aprendizaje y la distribución planificada de los contenidos disciplinares en función de su adecuación al momento formativo del estudiante.

En el tercer modelo curricular, el aprendizaje basado en problemas (ABP), la estrategia básica se centra en la metodología de aprendizaje. En cuanto a estructura curricular, el resultado es un currículo de contenidos mucho más integrados que los anteriores.

El ABP como estrategia curricular surge en 1969 en la Universidad de McMaster (Hamilton, Ontario, Canadá) y se extiende por todo el mundo en muy pocos años: Universidad de Maastricht (Países Bajos), Universidad de Newcastle (Australia), Universidad de Nuevo México (Estados Unidos), Universidad Ben Gurion (Israel)...

En esencia, el ABP consiste en facilitar al estudiante el aprendizaje de los contenidos y la adquisición de las competencias enfrentándolo a situaciones reales con incidencia en la salud, a partir de las cuales debe realizar un análisis de la situación y diseñar posibles soluciones a los problemas planteados seleccionando el material educativo a su alcance. En este modelo, el rol del profesor consiste en diseñar las situaciones, supervisar el proceso, aportar los materiales de aprendizaje, realizar la evaluación, fomentar el razonamiento del estudiante mediante técnicas de gestión del trabajo en grupos, tutorizar, etc., pero en ningún caso se convierte en el tradicional proveedor sistemático y dogmático de información.

Este modelo supone un cambio radical en todos los aspectos de la actividad formativa y requiere un gran cambio del currículo, de los recursos empleados y de la cultura del profesorado. El ABP constituye la alternativa más radical al tradicional modelo flexneriano, lo que ha propiciado numerosos estudios comparativos entre modelos [13].

Con frecuencia, la resolución de problemas como metodología docente también se utiliza satisfactoriamente como una actividad de aprendizaje en currículos no estructurados como tal.

La diferencia principal entre el modelo tradicional y el modelo de ABP consiste en que el primero centra su actividad en la transmisión de conocimientos y su acumulación por parte del estudiante, en tanto que el ABP la centra en la adquisición de estrategias y hábitos de razonamiento para afrontar las situaciones propias de la profesión.

El más reciente de los modelos curriculares contemplados se desarrolló en la Facultad de Medicina de la Universidad de Calgary (Alberta, Canadá) en 1995 [14]: es el denominado currículo por presentaciones clínicas. Este modelo, de diseño curricular integrado, utiliza metodología del ABP y se basa en el principio de intentar transferir al discente los puntos clave del razonamiento profesional, al objeto de que los pueda utilizar como modelo de razonamiento en el momento de enfrentarse a la realidad profesional. Para ello, y a diferencia del ABP, que parte de situaciones de la vida diaria, se define un número limitado de 'presentaciones' (razones por las que el paciente acude al médico) más frecuentes o relevantes. Ellas constituirán las unidades formativas a partir de las cuales se diseña el proceso de aprendizaje (objetivos y competencias, diagramas de decisión, objetivos de formación y actividades de aprendizaje), en el cual se mezclan discusiones de problemas con aproximaciones más dirigidas (prácticas y simulaciones). Las presentaciones pueden agruparse por razones organizativas. La Universidad de Brown, en Estados Unidos, está desarrollando un currículo basado en pacientes virtuales que constituye un paso más en la línea iniciada por la Universidad de Calgary. 


\section{Marco normativo de Bolonia}

El entorno normativo constituye en España uno de los principales puntos de partida del diseño curricular y condiciona las decisiones que se tomen acerca del modelo y diseño del nuevo currículo.

En el ámbito europeo, en lugar de un desarrollo legislativo específico, el proceso de Bolonia se está llevando a cabo en base a un conjunto de acuerdos, informes y recomendaciones emanados de comisiones ad hoc y refrendados por ministros europeos de educación en reuniones bianuales (Praga 2001, Berlín 2003, Bergen 2005, Londres 2007, Lovaina 2009, Budapest 2010). La propia Declaración de Bolonia [15], en que se fundamenta el proceso, constituye todo un ejemplo de sobriedad normativa (Tabla V).

Para el desarrollo del acuerdo sobre el modelo común de titulaciones, la Comisión Europea promovió el proyecto Tuning I [16], en cuyo seno se desarrolló el concepto de armonización, considerando que no es posible ni deseable homogeneizar los procesos formativos, que en gran parte deben quedar a la pericia de cada universidad; el modelo de reconocimiento debe basarse en las competencias adquiridas, que caracterizan los perfiles profesionales propios de los titulados, asegurando así la comparación de las capacidades de éstos.

Medicina no participó de ninguno de los proyectos Tuning (I y II), siendo diseñado a la medida de la titulación el proyecto MEDINE (Medical Education in Europe) [17], que ha asumido el papel armonizador desde parecidos principios.

En España, con anticipación al desarrollo legislativo, diversas instituciones pusieron en marcha proyectos para orientar el proceso de diseño curricular una vez se llevara a cabo el proceso legislativo regulador. El primero fue el proyecto Disseny [18], de la AQUCatalunya. seguido por el Libro blanco de la titulación de Medicina [19], elaborado bajo los auspicios de la Agencia Nacional de Evaluación de la Calidad y Acreditación (ANECA) por una comisión de la Conferencia de Decanos de las Facultades de Medicina Españolas (CNDFM) [20]. En ambos casos se definieron las competencias a adquirir como punto de partida común del proceso de planificación, basándose en la enumeración de las competencias básicas comunes del médico, elaboradas por expertos del International Institute of Medical Education (IIME) y descritas en el documento Global Minimum Essential Requirements (GMER) $[21,22]$. La versión del 'libro blanco' ha adquirido además valor normativo al ser incorporada por la Orden ECI/332/2008 [23] y, en consecuencia, cons-
Tabla V. Acuerdos que conforman la Declaración de Bolonia.

Creación de un modelo común de titulaciones reconocible por todos los estados

Adopción de un patrón de planificación (esquema bachelor/master o grado/posgrado)

Adopción del crédito ECTS como unidad de medida transferible del esfuerzo de aprendizaje

Armonización de los criterios de calidad

Eliminación de los obstáculos a la movilidad

tituye la base de las competencias incorporadas en todos los proyectos curriculares españoles.

El desarrollo legislativo estatal fue largo y complejo hasta la gestación de la Ley Orgánica 4/2007 [24], de 12 de abril, que modificaba la Ley Orgánica de Universidades del año 2001, adaptando el marco normativo universitario a los requerimientos del EEES. El desarrollo legislativo posterior fue rápido (el Real Decreto 1393/2007 [25], que concreta el marco de planificación de las nuevas titulaciones, se publicó el 29 de octubre del mismo año, y la orden que determina los requisitos para la verificación del título de médico, en febrero de 2008), aunque no lo suficiente para que las facultades cumplieran el objetivo de armonizarse en el 2010.

El marco de diseño curricular que se define en el Real Decreto 1393/2007 constituye un cambio copernicano respecto a las dos reformas anteriores: la de 1974, que instauró un plan de estudios basado en disciplinas de referencia para la titulación en todo el Estado, y la de 1992, que supuso una apertura del marco de diseño curricular que no todas las facultades aprovecharon en igual medida. El punto clave lo constituyó la ruptura de la unidad curricular del Estado, diversificando las formas en que se podían combinar los contenidos esenciales en las diferentes disciplinas, lo que facilitó la aparición de algunas integraciones horizontales. Sin embargo, en el esquema básico de planificación de 1992 se continuaba definiendo las materias de que debían componerse todos los planes de estudios, a través de un decreto de directrices específicas de cada titulación (Tabla VI).

Por contraposición, el modelo de 2007 ofrece libertad a las universidades para el diseño de sus propios currículos, con dos limitaciones: la orden ECI, que define los contenidos mínimos para titulaciones que, como Medicina, capacitan para el ejercicio de una profesión regulada, y la evaluación ex ante o verificación, instrumentada por el Consejo de Uni- 
Tabla VI. Características del diseño curricular en los sucesivos marcos legales españoles.

\begin{tabular}{lccc}
\hline & 1974 & 1992 & 2007 \\
\hline Troncalidad & Definida por asignaturas & Definida por materias & $\begin{array}{c}\text { Definida por contenidos } \\
\text { y competencias }\end{array}$ \\
\hline Optatividad (para el estudiante) & Nula & Elevada (optativas + libre elección) & Baja \\
\hline Materias preestablecidas & Todas & $\begin{array}{c}\text { Troncales } \\
\text { (grandes bloques disgregables) }\end{array}$ & Básicas de rama \\
\hline Practicum & No consta & Opcional & Obligatorio \\
\hline Integrabilidad & No posible & Horizontal & Global \\
\hline Oficialización & Reproducción del modelo & Adaptación a la normativa & $\begin{array}{c}\text { Coherencia del diseño y recursos } \\
\text { y cumplimiento de las directrices }\end{array}$ \\
\hline
\end{tabular}

versidades a través de la ANECA, paso imprescindible para la inscripción de la titulación en el Registro de Universidades, Centros y Titulaciones, que le confiere carácter oficial.

Dado que algunos de los acuerdos del Tratado de Bolonia forman ya parte de la estructura o los procedimientos de la mayor parte de las facultades españolas, sólo dos acuerdos suponen cambios mayores para que las facultades españolas puedan adaptarse al EEES: el reconocimiento de las titulaciones basadas en competencias y el relativo al sistema de calidad.

El concepto más disruptivo que ha aportado el proceso de Bolonia ha sido el de diseñar, aplicar y evaluar un currículo centrado en la adquisición de competencias que supera en mucho la complejidad del diseño dirigido a la adquisición de conocimientos.

Por lo que respecta a los procedimientos relacionados con la garantía de la calidad, en base a las indicaciones de la European Association for Quality Assurance in Higher Education (ENQA) [26] y de las agencias nacionales (ANECA [27] en España), se ha puesto en marcha un complejo sistema de verificaciones y acreditaciones que obliga a las universidades a una planificación exhaustiva de sus titulaciones y a realizar un esfuerzo de transparencia y coherencia con los planes elaborados y con las competencias a conseguir, que además de exigir un importante esfuerzo organizativo, implica un replanteamiento tanto ideológico como metodológico y de recursos para la formación, así como la implementación de un sistema interno de gestión de la calidad (SGIC). Todo ello conlleva unos requerimientos de documentación inusuales en la cultura académica española. En este sentido, cabe esperar que el esfuerzo realizado para la evaluación ex ante y el seguimiento de las titulaciones (programa Verifica de ANECA) se corresponda con uno similar para la evaluación ex post (programa Monitor de ANECA) del cumplimiento de los planes aprobados.

\section{Situación actual}

La elaboración de los nuevos currículos adaptados a los Reales Decretos 1393/2007 y 861/2010 [28] y la Orden ECI 332/2008 marcan el inicio de un largo proceso que ha de suponer la actualización de los procesos formativos de los médicos en España de acuerdo con las premisas marcadas por los acuerdos que conforman el EEES. A partir de este laxo corsé legal, el proceso de diseño se lleva a cabo influido por fuerzas que imprimen un ajuste más preciso y condicionan aspectos muy relevantes del resultado final. Una de ellas es el modelo de titulación que se desprende de la literalidad del 'libro blanco' de ANECA/CNDFM, en general poco comprometido con modelos y estrategias educativos, pero con una orientación compatible con el desarrollo de una educación médica centrada en la dimensión profesional. No hay duda posible respecto a la influencia de dicha publicación en los currículos elaborados por los grupos de trabajo de las facultades. La segunda lo constituye el proceso de verificación de los títulos, que exige que el diseño contemple, con un mínimo de coherencia, la definición de los resultados de formación y de las competencias, así como una pormenorizada descripción de las metodologías de aprendizaje y de evaluación. 
Finalmente, aun cuando la inercia tecnológica y cultural de las instituciones sea un componente mayor de las fuerzas que guían los nuevos diseños, numerosos currículos incluyen referencias a las recomendaciones de las organizaciones nacionales e internacionales (SEDEM, AMEE, IIME, WFME, etc.) que influyen por lo menos en el lenguaje de planificación, y que con el tiempo pueden convertirse en catalizadores de un necesario cambio de modelo, una aportación significativa de Bolonia a la educación médica en España [29].

Los planes de estudios elaborados en España para la adaptación al EEES responden, con un par de excepciones, a un modelo curricular flexneriano evolucionado, sin que aparezca prácticamente ninguna propuesta integrada ni basada en problemas o en presentaciones clínicas.

La evolución del modelo se pone en evidencia por propuestas que contienen ejemplos de integración horizontal, con frecuencia de carácter táctico (para ajustar tamaños de materias a una modularización preestablecida), o por variaciones en el nombre de las materias, que en ocasiones reflejan intenciones de reorientación de los contenidos. Algunas propuestas incluyen materias de introducción precoz en la clínica o materias de integración con un número de créditos muy bajo que pretenden dotar al estudiante de una visión integrada de las materias.

El cambio real inducido por la introducción de los módulos como estructura que agrupa diferentes materias o asignaturas ha sido poco significativo $y$, en parte, negativo. Poco significativo porque en ningún caso se ha concedido estructura organizativa alguna al módulo, siendo la materia la unidad de programación y de evaluación, con lo que el módulo no va a tener entidad real en el desarrollo operativo del programa, y negativo porque el propio criterio de definición de los módulos ha contribuido a alejar las posibilidades de organización de aquéllas desde un criterio de integración vertical.

Tampoco la introducción del crédito ECTS, que valora la actividad no presencial del estudiante, facilitando así un planteamiento de la formación centrada en el aprendizaje, parece haber logrado el objetivo deseado de cambiar una estrategia de planificación definida por la función del que enseña por otra definida por la actividad del que aprende. La maniobra de considerar el crédito ECTS de 25 horas como 10 horas presenciales más 15 no presenciales para todos las materias ha asimilado rápidamente el crédito ECTS al crédito tradicional, con lo que la planificación de actividades se centra en las actividades presenciales e ignora las no presenciales.
Tabla VII. Innovaciones relevantes aportadas por los currículos adaptados al Espacio Europeo de Educación Superior.

\begin{tabular}{ll}
\hline Aportación de nuevos contenidos & Poco frecuente \\
\hline Integración horizontal de contenidos & Frecuente \\
\hline Definición de competencias & Generalizado \\
\hline Definición de resultados de aprendizaje & Generalizado \\
\hline Contacto precoz con la clínica & Poco frecuente \\
\hline Materias de integración & Ocasional \\
\hline Actividades en grupo reducido (seminarios, etc.) & Generalizado \\
\hline Elaboración de trabajos & Muy frecuente \\
\hline Actividades en aulas de actividades clínicas & Frecuente \\
\hline Rotaciones clínicas & Generalizado \\
\hline Rotaciones en el ámbito de la medicina primaria & Frecuente \\
\hline Evaluaciones complejas (más que un examen) & Muy frecuente \\
\hline Elínica objetiva estructurada & Frecuente \\
\hline Frecuente
\end{tabular}

Respecto a las actividades de aprendizaje previstas, elemento básico en la definición de un modelo formativo y clave para poder valorar la aproximación del currículo real al formal, éstas se ven muy condicionadas por el hecho de que la maquinaria académica de las universidades se ha encargado de uniformizar un porcentaje preestablecido de actividades en grupo grande (clases magistrales), mediano o pequeño, asegurándose así que el incremento de actividades en grupos reducidos no va a suponer un incremento de las necesidades de plantilla. Este hecho se explica por la reticencia de los equipos de gobierno a la toma de decisiones discriminatorias hacia titulaciones con una mayor exigencia metodológica, pero no es menos cierto que esta normativa (generalizada) ha contribuido a desmotivar a las facultades de medicina a proponer modelos curriculares diferentes del tradicional, como exigiría, por ejemplo, un currículo diseñado por problemas. Tampoco debería ignorarse lo poco justificable pedagógicamente que puede resultar enfrentarse al diseño de una asignatura con unas cuotas cerradas 
de tipos de actividades, condicionadas por las cuotas de tamaños de grupo.

Más allá del modelo curricular como elemento primario, dos grandes paradigmas que ha aportado la puesta en marcha del EEES han influido con fuerza en el diseño de los nuevos planes de estudio: la orientación de los planes hacia los resultados de aprendizaje y la necesidad de concretar las competencias a adquirir durante el programa de formación. La capacidad de orientar el diseño de currículos por parte de ambos elementos más allá de la ortodoxia de aplicación de un modelo está bien reflejada en la bibliografía [30-32] como una poderosa estrategia para la orientación de decisiones metodológicas y de previsión de recursos para la construcción de un currículo. De hecho, el planteamiento de unos resultados centrados en la actividad profesional y el diseño de las actividades más adecuadas para conseguirlos constituyen las aportaciones más relevantes para la innovación curricular.

Afortunadamente, en este sentido, tanto las orientaciones ideológicas de la convergencia europea (Tuning, MEDINE, 'libro blanco,' Disseny, etc.) como el desarrollo normativo y el propio sistema de verificación han coincidido en el papel central de los resultados de formación y las competencias del título como elemento definitorio de éste. Tal orientación se concreta claramente en los requisitos para la verificación del título, en el que no sólo deben concretarse los objetivos, sino también las competencias a adquirir y los resultados de formación, y se valora la coherencia de los diferentes aspectos del diseño curricular y de los recursos previstos para su desarrollo. Esta orientación del marco normativo y su traslación al sistema de evaluación ha tenido efectos importantes sobre el diseño curricular llevado a cabo por las facultades, algunas de las cuales ya contaban con efectivos sensibilizados hacia esta revolución conceptual, claramente contrapuesta a la mera actualización o reorganización de contenidos.

Así, de forma generalizada, los nuevos currículos prevén una mayor variedad de metodologías docentes para el desarrollo del aprendizaje. Cabe destacar el hecho de que numerosos currículos prevén el uso de las aulas de habilidades para la formación. También merece destacarse la generalización de periodos de estancias clínicas, en general en el último curso, que suponen un promedio de unas 1.000 horas de experiencia clínica en el momento de la titulación.

Por lo que respecta a las estrategias de evaluación -elemento crucial de la formación que puede dar, más que ningún otro, la medida de los cambios que se pueden producir en el aprendizaje de los estudiantes-, en general se contemplan metodologías múltiples de evaluación para cada materia que incluyen, en el caso de muchas materias clínicas, la evaluación clínica objetiva estructurada (ECOE), y el uso de portafolios de aprendizaje en la evaluación de las estancias clínicas.

Las aportaciones planteadas en los nuevos currículos adaptados al EEES, aun sin abandonar el modelo curricular flexneriano, suponen sobre el papel mejoras importantes en el desarrollo de los programas de formación y permiten enfocar el proceso de cambio con un moderado optimismo. El compromiso del profesorado con los nuevos diseños y su capacidad para llevarlos a cabo serán, finalmente, la clave para asegurar el éxito de la reforma.

\section{Bibliografía}

1. Harden RM. Curriculum mapping; a tool for transparent and authentic teaching and learning. AMEE Guide no. 21. Med Teach 2001; 23: 123-37.

2. English FW. Quality control in curriculum development Arlington, VA: American Association of School Administration; 1978.

3. Shewart W. Statistical method from the viewpoint of quality control. Washington: Graduate School, Department of Agriculture; 1939.

4. Álvarez X, Geli AM, Pedró F, Prades A, Prat J, Rodríguez S, et al. Marc general per al disseny, el seguiment i la revisió de plans d'estudis i programes. Barcelona: Agència per a la Qualitat del Sistema Universitari a Catalunya; 2002.

5. Prideaux DJ. Curriculum development in medical education: from acronyms to dynamism. Teaching and Teacher Education 2007; 23: 294-302.

6. Papa FJ, Harasym PH. Medical curriculum reform in North America, 1765 to the present: a cognitive science perspective. Acad Med 1999; 74: 154-64.

7. Prat J, Oriol A. Nuevas orientaciones en los programas universitarios de preparación de profesionales médicos. Barcelona: Fundación Medicina y Humanidades Médicas; 2004.

8. Harden RM, Sowden S, Dunn WR. Educational strategies in curriculum development: the SPICES model. Med Educ 1984. 18: 284-97.

9. Barrow M, McKimm J, Samarasekera D. Strategies for planning and designing medical curricula and clinical teaching. South East Asian Journal of Medical Education 2010; 4: 2-8.

10. Bligh J, Prideaux D, Parsell G. PRISMS: new educational strategies for medical education. Med Educ 2001; 35: 520-1.

11. Flexner A. Medical education in the United States and Canada. New York: The Carnegie Foundation for the Advancement of Teaching; 1910.

12. Jippes M, Majoor GD. Influence of national culture on the adoption of integrated and problem-based curricula in Europe. Med Educ 2008; 42: 279-85.

13. Albanese MA, Mitchell S. Problem-based learning: a review of literature on its outcomes and implementation issues. Acad Med 1993; 68: 52-81.

14. Mandin M, Harasym H, Harasym P, Eagle C, Watanabe M Developing a 'clinical presentation' curriculum at the University of Calgary. Acad Med 1995; 70: 186-93.

15. El espacio europeo de la enseñanza superior. URL: http:// www.crue.org/export/sites/Crue/procbolonia/documentos/ antecedentes/2._Declaracixn_de_Bolonia.pdf.

16. González J, Wagenaar R. Tuning educational structures in Europe. Bilbao: Universidad de Deusto; 2003. 
17. MEDINE2. URL: http://www.medine2.com.

18. Prat J, Carreras J, Branda L, Miralles R, Fenoll MR, Rodríguez $\mathrm{S}$, et al. Competències professionals bàsiques comunes dels llicenciats en Medicina formats a les universitats de Catalunya. Barcelona: Agència per a la Qualitat del Sistema Universitari de Catalunya; 2004.

19. Agencia Nacional de Evaluación de la Calidad y Acreditación. Libro blanco. Título de Grado en Medicina. URL: http:// www.aneca.es/var/media/150312/libroblanco_medicina_ def.pdf.

20. Conferencia Nacional de Decanos de Facultades de Medicina. URL: http://www.cndmedicina.com.

21. Comité Central del Institute for International Medical Education. Requisitos globales mínimos esenciales en educación médica. Educ Med 2003; 6 (Supl 2): 11-9.

22. Institute for International Medical Education. URL: http:// www.iime.org.

23. Orden ECI/332/2008, de 13 de febrero. Boletín Oficial del Estado 2008; 40: 8351-5.

24. Ley Orgánica 4/2007, de 12 de abril. Boletín Oficial del Estado 2007; 89: 16241-60.
25. Real Decreto 1393/2007, de 29 de octubre. Boletín Oficial del Estado 2007; 260: 44037-48.

26. European Association for Quality Assurance in Higher Education. URL: http://www.enqa.eu.

27. Agencia Nacional de Evaluación de la Calidad y Acreditación URL: http://www.aneca.es.

28. Real Decreto $861 / 2010$, de 2 de julio. Boletín Oficial del Estado 2010; 161: 58454-68.

29. Arnalich-Fernández F. Adaptación del nuevo grado de medicina al Espacio Europeo de Educación Superior, ¿cuál ha sido la aportación de Bolonia? Educ Med 2010; 13 (Supl 1): S3-7.

30. Smith SR, Dollase RH. Planning, implementing and evaluating a competency-based curriculum. Med Teach 1999; 21: 5-22.

31. Royal College of Physicians and Surgeons of Canada. URL: http://rcpsc.medical.org/canmeds/index.php.

32. Harden RH, Crosby JR, Davis MH, Smith SR, Dollase R, Friedman Ben-David M, et al. Outcome-based education. AMEE Education Guides no. 14; 1999 